\title{
Comparison of AC/A Ratio in True and Simulated Divergence Excess Exotropia
}

\author{
Sana Sagheer ${ }_{1,2,5}$, Muhammad Ali Haider ${ }^{2}$, Attaullah Shah Bukhari ${ }^{3}$, Uzma Sattar ${ }^{4}$, Iftikhar Ahmed ${ }^{5}$ \\ ${ }_{1,2,4,5}$ Punjab Rangers Teaching Hospital/Rahbar Medical \& Dental College, Lahore \\ ${ }^{3}$ Khairpur Medical college, Khairpur Mirs
}

\section{ABstRact}

Purpose: To compare the accommodation convergence per accommodation ratio $(\mathrm{AC} / \mathrm{A})$ in true and simulated divergence excess exotropia.

Study Design: Comparative cross sectional study.

Place and Duration of Study: Department of ophthalmology, Punjab Rangers teaching hospital, Lahore, from July 2019 to December 2019.

Methods: The study included 20 patients each of intermittent exotropia (XT) true and simulated divergence excess exotropia (XT).All the subjects underwent complete orthoptic assessmentthat included AC/A ratio calculation through heterophoria method after monocular occlusion.

Results: The Results showed that patients with true divergence excess exotropia have a high AC/A ratio as compared to patients with simulated divergence excess exotropia who have a normal AC/A ratio. 57\% patients showed true divergence excess exotropia with high AC/A ratio while $43 \%$ had divergence excess with normal AC/A ratio.

Conclusion: The response of $\mathrm{AC} / \mathrm{A}$ was found to be higher in patients with true divergence excess exotropia after monocular occlusion.

Key Words: Intermittent exotropia, True simulated divergence excess exotropia, simulated divergence excess exotropia.

How to Cite this Article: Sagheer S, Haider MA, Bukhari AS, Sattar U, Ahmed I. Comparison of AC/A Ratio in True and Simulated Divergence Excess Exotropia. Pak J Ophthalmol. 2021, 37 (2): 168-172.

Doi: http://doi.org/10.36351/pjo.v37i2.1192

\section{INTRODUCTION}

The control of squint depends upon different factors such as the distance of fixation fatigue, bright light, concentration and ill health. ${ }^{1}$ In Intermittent exotropia, eyes remain straight with binocular single vision at

Correspondence: Muhammad Ali Haider

Department of Ophthalmology, Rahbar Medical \& Dental College, Lahore

Email: alihaider_189@yahoo.com

Received: January 3, 2021

Accepted: February 14, 2021 some times and manifest suppression at other times. Presentation is usually around age of 2 years with exophoria which breaks down to tropia under above mentioned circumstances. ${ }^{2}$ In distance exotropia, deviation of eyes is greater at distance then near and increases further beyond 6 meters. It is further classified into two as true divergence excess exotropia and simulated divergence excess exotropia. ${ }^{3}$

In true divergence excess exotropia the distance deviation is greater than near deviation before and after patch test. The difference of distance and near is almost 10 prism diopters. In cases of simulated divergence excess exotropia, the near deviation equals distance deviation after patch test of 30 minutes. ${ }^{4}$ Patch test differentiate between true divergence and 
simulated divergence excess exotropia. Monocular occlusion eliminates the tenacious proximal fusion. ${ }^{5}$

All the cases of divergence excess exotropia donot have true divergence excess exotropia.Monocular occlusion of 30 to 60 minutes reveals large deviation at near in cases of simulated divergence exotropia. ${ }^{6}$ When a patient shows large deviation at near after monocular occlusion test it is called tenacious proximal fusion and when a patient shows a small deviation after prolonged patch test this is called high AC/A ratio. But when patients show same amount of deviation after patch of 3D lenses, it is called proximal convergence, which is True Convergence Excess. ${ }^{7}$

$\mathrm{AC} / \mathrm{A}$ ratio are the amount of accommodative convergence induced by each diopters of accommodation exerted and is measured in prism diopters. Normal range of AC/A ratio is 2-4. The relationship of AC/A ratio is fixed and remains unchanged from childhood till the age of presbyopia. The AC/A ratio can be modified permanently by surgery of the extra ocular muscles and temporarily by drugs and lenses. ${ }^{8}$ Different methods are used to measure AC/A ratio; heterophoria method, gradient method, fixation disparity method and graphical method. Most common method is heterophoria method in which a prism cover test is performed for distance and near fixation .Inter-pupillary distance is measured. A positive sign is used for esotropia and negative sign is used for exotropia.

$$
\text { AC/A ratio }=\text { IPD }(\text { in } \mathrm{cm})+\frac{\text { Near PCT }- \text { distance PCT }}{\text { Amount of accom. Exerted }}
$$

The patch test is used to control the tonic fusional convergence to differentiate pseudo-divergence excess from true divergence excess and to reduce the angle variability. Contrary to the earlier practice of patching one eye for 24 hours, it is now found that patching the eye for 30 minutes is sufficient to suspend the tonic fusional convergence and thus reveals the actual amount of deviation. ${ }^{9}$ Comparison of distance and near deviation is important part of evaluation in strabismus. In majority of esotropic patients, the difference of distance and near deviation shows a normal linkage of convergence and accommodation and this relation can be explained by AC/A ratio. In cases of intermittent exotropia it is different because many are slow to dissipate proximal fusion mechanism which prevents them from manifesting true near deviation during a brief cover test. ${ }^{10}$ If a patch test is not performed in cases of intermittent exotropia before surgery they may develop cyclic esotropia with high AC/A ratio. ${ }^{11}$

Rationale of the study was to compare the accommodative convergence per unit accommodation ratio $(\mathrm{AC} / \mathrm{A})$ in true and simulated divergence excess exotropia in Pakistani patients.

\section{METHODS}

In this analytical, comparative cross sectional study, patients with divergence excess exotropia presenting in the orthoptics clinic of ophthalmology department of Punjab Rangers Teaching Hospital, Rahbar Medical and Dental College were enrolled. Patients with constant and basic type of deviations were excluded. The minimum age chosen for study was 5 years and maximum 30 years to ensure maximum cooperation. Examination included Visual acuity (VA) record by using Log MAR chart.VA was recorded in adults using alphabets. In young children using picture charts and in illiterates with tumbling $\mathrm{E}$ chart. All the subjects underwent complete orthoptic assessment and cycloplegic refraction when required. AC/A ratio were calculated using heterophoria method. For all measurements, patient and observer were at same eye level with good room illumination. For distance measurements, patient was seated at 6meters. Fixation target at Log MAR chart was 2 lines above the best corrected visual acuity to maintain accurate fixation. Patient was asked to fixate at a distant target and prism was placed in front of deviated eye. Alternate prism cover test was performed. Prism power was increased until there was no movement of eyes to take up fixation. Amount of deviation at distance was calculated. A patch test of 30 minutes was performed in each patient. One eye was occluded for half an hour, deviation was measured again for near and any change in deviation was noted. Inter-pupillary distance was measured by instructing the patient to see straight ahead.AC/A ratio was calculated by formula described above. All the collected data was noted and analyzed by using SPSS 22 version.

\section{RESULTS}

AC/A ratio was high in true divergence excess exotropia $(28.21 \%)$ and normal in simulated divergence excess exotropia (13.19\%) Table 1. Table 2 shows that there was significant difference between true and pseudo divergence excess exotropia. AC/A ratio was normal in cases of simulated divergence 
Table 1: Group Statistics.

\begin{tabular}{lllrcr}
\hline & Type of Deviation & N & Mean & Std. Deviation & Std. Error Mean \\
\hline Accommodative convergence & True divergence excess & 23 & 11.0739 & 1.93537 & 0.40355 \\
laccommodation & Simulated & 17 & 5.1765 & 0.24630 & 0.05974 \\
\multirow{2}{*}{ Interpupillary distance } & True divergence excess & 23 & 5.3913 & 0.47569 & 0.09919 \\
& Simulated divergence excess & 17 & 5.1765 & 0.24630 & 0.05974 \\
\hline
\end{tabular}

Table 2: Independent Samples Test.

\begin{tabular}{|c|c|c|c|c|c|c|c|c|c|c|}
\hline & & \multicolumn{2}{|c|}{$\begin{array}{c}\text { Levene's Test } \\
\text { for Equality of } \\
\text { Variances }\end{array}$} & \multicolumn{7}{|c|}{ t-test for Equality of Means } \\
\hline & & \multirow[t]{2}{*}{$\mathbf{F}$} & \multirow{2}{*}{ Sig. } & \multirow[t]{2}{*}{$\mathbf{T}$} & \multirow{2}{*}{ Df } & \multirow{2}{*}{$\begin{array}{c}\text { Sig. } \\
\text { (2-tailed) }\end{array}$} & \multirow{2}{*}{$\begin{array}{c}\text { Mean } \\
\text { Difference }\end{array}$} & \multirow{2}{*}{$\begin{array}{l}\text { Std. Error } \\
\text { Difference }\end{array}$} & \multicolumn{2}{|c|}{$\begin{array}{l}\text { 95\% Confidence Interval of } \\
\text { the Difference }\end{array}$} \\
\hline & & & & & & & & & Lower & Upper \\
\hline \multirow{2}{*}{$\mathrm{AC} / \mathrm{A}$} & $\begin{array}{l}\text { Equal variances } \\
\text { assumed }\end{array}$ & 15.910 & 0.000 & 12.448 & 38 & 0.000 & 5.89744 & 0.47377 & 4.93835 & 6.85654 \\
\hline & $\begin{array}{l}\text { Equal variances not } \\
\text { assumed }\end{array}$ & & & 14.456 & 22.959 & 0.000 & 5.89744 & 0.40795 & 5.05345 & 6.74143 \\
\hline \multirow{2}{*}{$\begin{array}{l}\text { Inter-pupillary } \\
\text { distance }\end{array}$} & $\begin{array}{l}\text { Equal variances } \\
\text { assumed }\end{array}$ & 30.268 & 0.000 & 1.698 & 38 & 0.098 & .21483 & 0.12655 & -0.04136 & 0.47102 \\
\hline & $\begin{array}{l}\text { Equal variances not } \\
\text { assumed }\end{array}$ & & & 1.855 & 34.595 & 0.072 & .21483 & 0.11579 & -0.02033 & 0.44999 \\
\hline
\end{tabular}

excess exotropia and high $\mathrm{AC} / \mathrm{A}$ ratio was recorded in true divergence excess exotropia. $(\mathrm{t}=12.448$, df 38 , $\mathrm{p}=0.000)$.

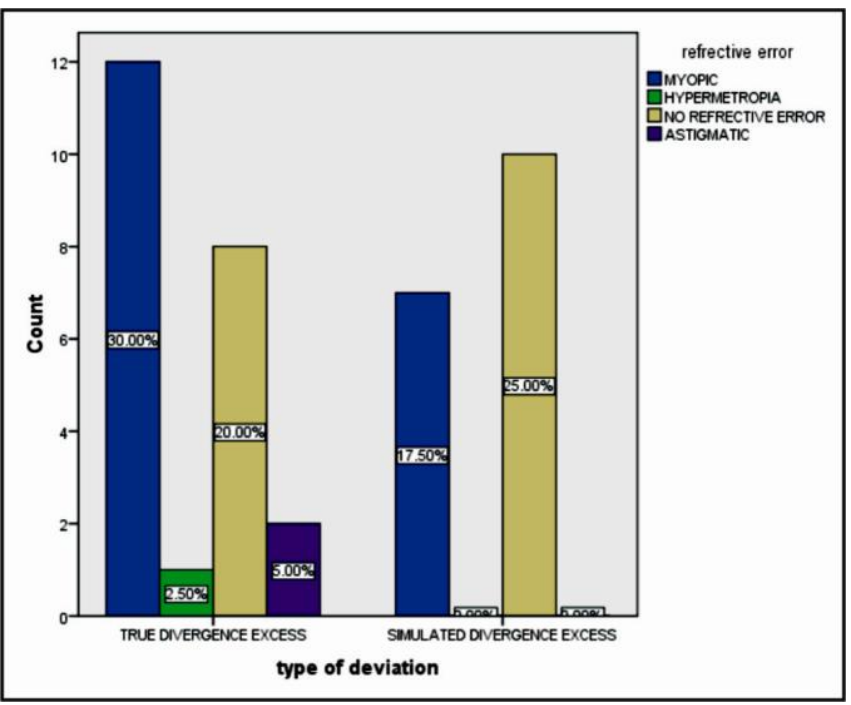

This graph shows that most of the patients of true and simulated divergence excess exotropia were myopic or without refractive errors. Thirty percent of patients were myopic in true divergence excess exotropia and $17.50 \%$ were myopic in simulated. In both groups $20 \%$ to $25 \%$ were emmetrope.

\section{DISCUSSION}

The study was designed to differentiate the cases of true divergence excess exotropia from simulated divergence excess exotropia by performing a patch test and measuring AC/A ratio by herterophoria method. Apparently both types of cases show high AC/A (accommodative convergence per unit accommodation) ratio prior to patch test but post monocular occlusion test the results are different.Patch test reveals the real amount of deviation at near. ${ }^{12}$ It is generally believed that AC/A ratio was found higher in divergence excess exotropia type. Over $75 \%$ of patient have increase in near deviation after patch test and decrease in calculated AC/A ratio. ${ }^{13}$

By using standard methods, Cooper and Coworkers calculated AC/A in patients of intermittent exotropia. Patient first underwent a monocular occlusion of one hour for measurement of actual near deviation. ${ }^{14,15}$ If the patient had high $\mathrm{AC} / \mathrm{A}$ ratio before occlusion test but had normal AC/A ratio after monocular occlusion test they described these cases as pseudo high AC/A ratio. If tenacious proximal fusion 
was not suspended, the near measurements were contaminated in patients of exotropia. ${ }^{16,17}$

In addition to the factors enumerated by Scobee to explain this phenomenon, we believe that extremely active convergence tonus during childhood may be a factor in obscuring the exodeviation at near fixation. This mechanism enables patients with a basic deviation to keep their eyes aligned for near vision but not for distance vision, where convergence is less active. Momentary disruption of fusion by alternately covering each eye rapidly in prism cover test or alternate cover test is not enough to break the compensatory mechanism that have been developed in years. Kushner introduced the term "tenacious proximal fusion" for the persistent convergence innervation that hides the exodeviation at near fixation. This term seems awkward and does not add to the clarification of the issue. The term "convergence aftereffect" is a more appropriate description of this phenomenon. $^{18,19}$

In another study which was conducted to evaluate the diagnostic method of divergence excess exotropia, 237 patients were evaluated. They were divided into two groups according to their response to monocular occlusion and the use of +3.00 diopters add at near. They observed that in ten patients there was increase in deviation in both tests. They considered these cases as simulated divergence excess exotropia and ten patients who did not respond to monocular occlusion and remained unchanged, these were the True divergence excess exotropia patients. ${ }^{20}$

To review the multiple factors responsible for the discrepancy in distance and near deviation, two clinical tests (occlusion test and +3.00 diopter lens) were described. Patients with intermittent exotropia usually have more deviation at distance as compared to near, and these cases have high AC/A ratio after occlusion of one eye and with the use of +3.00 spheres at near. ${ }^{18}$

\section{CONCLUSION}

The response of AC/A was found to be higher in patients with true divergence excess exotropia after monocular occlusion.

\section{Ethical Approval}

The study was approved by the Institutional review board/Ethical review board. (EC Ref No: 01/18).

\section{Conflict of Interest}

Authors declared no conflict of interest.

\section{REFERENCES}

1. Sheppard AL, Wolffsohn JS. Digital eye strain: prevalence, measurement and amelioration. BMJ Open Ophthalmol. 2018; 3 (1): e000146.

Doi: 10.1136/bmjophth-2018-000146. PMID: 29963645; PMCID: PMC6020759.

2. Jae-Wook Jung, Se-Youp Lee. A Comparison of the Clinical Characteristics of Intermittent Exotropia in Children and Adults. Korean J Ophthalmol. 2010; 24 (2): 96-100.

3. Audren F. Intermittent Exotropia. J Fr Ophtalmol. 2019; 42 (9): 1007-1019.

4. Horwood AM, Riddell PM. Evidence That Convergence Rather Than Accommodation Controls Intermittent Distance Exotropia. Acta Ophthalmol. 2012; 90 (2): 109-117.

5. Arnoldi KA, Reynolds JD. Diagnosis of PseudoDivergence Excess Exotropia Secondary to High Accommodative Convergence to Accommodation Ratio. Am Orthopt J. 2006; 56: 133-137.

6. Jung EH, Kim S, Yu YS. Comparison of the Characteristics of Patients with Intermittent Exotropia According to Response to Diagnostic Monocular Occlusion. JPN J Ophthalmol. 2018; 62 (2): 243-248.

7. Khawam E, Zein W, Haddad W, Haddad C, Allam S. Intermittent Exotropia with High AC/A Ratio: Is It a Bane to Surgical Cure? Some Facts and Fictions of the Two Clinical Tests: Occlusion of One Eye and the Use of +3.00 Spherical Lenses. Binocul Vis Strabismus Q. 2003; 18 (4): 209-216.

8. Park JW, Lee SJ. Comparison of Binocular Function and Surgical Outcomes of Tenacious Proximal Fusion and High Accommodative Convergence/ Accommodation Ratio Types of Intermittent Exotropia. Korean J Ophthalmol. 2018; 32 (6): 483-487.

9. Heo H, Sung MS, Park SW. Surgical Outcomes of Intermittent Exotropia with a High Accommodation Convergence-Accommodation Ratio. Invest Ophthalmol Vis Sci. 2013; 54: 3641.

10. Knapp P. Divergent deviations. In: Allen JH ed. Strabismic ophthalmic symposium II. St. Louis 1958, Mosby-Year Book 354.

11. Wang $\mathbf{X}$, Chen B, Liu L. Cyclic Esotropia with Development of a High Accommodative Convergence to Accommodation Ratio after Surgery for Intermittent Exotropia. Int Ophthalmol.2017; 37 (4): 1069-1072.

12. Han JM, Yang HK, Hwang J. Efficacy of Diagnostic Monocular Occlusion in Revealing the Maximum Angle of Exodeviation. Br J Ophthalmol. 2014; 98 (11): $1570-1574$. 
13. Le T, Koklanis K, Georgievski Z. The Fixation Target Influences the Near Deviation and AC/A Ratio in Intermittent Exotropia. J AAPOS. 2010; 14 (1): 25-30.

14. Cooper J, Medow N. Intermittent exotropia basic and divergence excess type. Binocular Vision Eye Muscle Surg Q. 1993; 8 (3): 185-216.

15. Niederker $\mathbf{O W}$. The value of diagnostic occlusion for intermittent exotropia. Am Orthopt J. 1974; 24: 69-72.

16. Khawam E, Zein W, Haddad W, Haddad C, Allam S. Intermittent exotropia with high AC/A ratio is it a bane to surgical cure? Some facts and fictions of the two clinical tests: occlusion of one eye and the use of +3.00 spherical lenses. Binocul Vis Strabismus. 2003; 18 (4): 209-216.

17. Arnoldi KA, Reynolds JD. Diagnosis of PseudoDivergence Excess Exotropia Secondary to High Accommodative Convergence to Accommodation Ratio. Am Orthopt J. 2006; 56 (1): 133-137.

18. Zhang KK, Koklanis K, Georgievski Z. Exotropia. A Review of the Natural History and Non-surgical Treatment Outcomes. Aus Orthopt J. 2007; 39 (1): 3137.

19. Frantz KA. The importance of multiple treatment modalities in a case of divergence excess. J Am Optom Assoc. 1990; 61 (6): 457-462.

20. Daum KM. Divergence excess: characteristics and results of treatment with orthoptics. Ophthalmic Physiol Opt. 1984; 4 (1): 15-24.

\section{Authors' Designation and Contribution}

Sana Sagheer; Orthoptist: Concepts, Design, Literature search, Data acquisition, Data analysis, Manuscript review.

Muhammad Ali Haider; Assistant Professor: Literature search, Manuscript preparation, Manuscript editing, Manuscript review.

Attaullah Shah Bukhari: Assistant Professor: Data analysis, Statistical analysis, Manuscript review.

Uzma Sattar; Investigative Occulist: Manuscript editing, Manuscript review.

Iftikhar Ahmed; Professor: Manuscript editing, Manuscript review. 